\title{
Mechanisms of Arterial Thrombosis in Nonparallel Streamlines: Platelet Thrombi Grow on the Apex of Stenotic Severely Injured Vessel Wall
}

\author{
Experimental Study in the Pig Model
}

\author{
L. Badimon and J. J. Badimon \\ Division of Cardiology, Department of Medicine, Mount Sinai School of Medicine, New York, New York 10029
}

\begin{abstract}
The role of thrombosis in various acute coronary syndromes has been established. However, the basic mechanism by which plaque rupture leads to a growing thrombus in the vicinity of stenotic lesions is not well understood. Using a characterized flow chamber in a rheologically controlled system, we have mimicked stenotic vessels and studied for the first time cellvessel wall interaction in nonparallel streamlines. Stenoses ranging from 0 to $80 \%$ were produced with stripped tunica media to mimic severe vessel wall damage, and perfused with heparinized flowing blood. This perfusion device was placed within an extracorporeal system in swine, and blood was perfused for selected times from 1 to $30 \mathrm{~min}$. Platelet deposition on the surface was evaluated by ${ }^{111}$ Indium-labeled platelets. As percent stenosis increased, platelet deposition significantly increased $(P<0.001)$, indicating a shear-induced cell activation. Analysis of the axial distribution of platelet deposition indicated that the apex, and not the flow recirculation zone distal to the apex, was the segment of greater platelet accumulation within $30 \mathrm{~min}$ of blood perfusion $(P<0.001)$. These results also indicate that the severity of the acute platelet response to plaque rupture probably depends on the location of the rupture with relation to the apex of the plaque.
\end{abstract}

\section{Introduction}

Thrombus formation resulting from plaque disruption or angioplasty may be related to several biochemical and physical factors that occur subsequent to the vascular trauma. Those factors are influenced by $(a)$ the nature of the flowing blood, (b) the exposure of different specific elements within the vessel wall, and $(c)$ changes in local flow conditions in the vicinity of the plaque.

Blood flow determines both the arrival and removal of cells and fluid-phase proteins from an exposed surface. Interactions occurring when blood contacts a reactive surface are controlled by transport processes in the bloodstream immediately above the surface and by reactions at the surface itself (1). Whether arrival or removal predominates for a given flowing cell is essentially regulated by its binding to the surface or to

Address reprint requests to Dr. Lina Badimon, Division of Cardiology, Annenberg 8-06/Box 1030, The Mount Sinai Medical Center, One Gustave L. Levy Place, New York, NY 10029.

Received for publication 4 November 1988 and in revised form 26 June 1989.

J. Clin. Invest.

(c) The American Society for Clinical Investigation, Inc.

$0021-9738 / 89 / 10 / 1134 / 11 \$ 2.00$

Volume 84, October 1989, 1134-1144 other cells relative to the shear forces imposed by the local flow patterns on the cells at the surface.

We have previously reported the important role of the perfused substrate in triggering platelet adhesion and thrombus growth in laminar flow conditions (2-5) and in in vivo models of carotid artery injury (6-8).

Prior studies using perfusion chambers have studied platelet-vessel wall interaction under controlled rheological conditions at low and high shear rates typical of patent or stenotic vessels, but all those studies have been performed in laminar flow conditions. In atherosclerotic vessels laminar flow conditions may not be maintained since stenotic narrowings induce flow disturbances that modify cell-cell and cell-vessel interactions and the local concentration of fluid-phase chemical mediators necessary for cell interaction.

This is the first controlled study of the acute platelet response to a severely damaged vessel wall in nonparallel streamline flow. The objective of this study was to evaluate the influence of increasing degrees of eccentric stenosis on the development and growth of platelet thrombus and on the axial distribution of cell aggregates. We found that platelet deposition on the damaged artery increased significantly with increasing stenosis. In addition, we found that platelet thrombi grew preferentially at the apex of the stenosis even though similarly damaged vessel wall was exposed in the segments with different local flow profiles. Finally, we observed that large thrombi have a tendency to be dislodged and that the residual mural thrombotic mass is extremely reactive to further platelet thrombosis.

Hence, this study suggests that the severity of the thrombotic complications of plaque rupture will depend on the location of the rupture with relation to the apex of the plaque.

\section{Methods}

Experimental conditions. The animal model for the study was the Yorkshire albino pig obtained from a single local farm (body wt, $35 \pm 3$ $\mathrm{kg}$ ). 50 normal pigs were randomly assigned to the various experimental protocols of the study. They were housed in facilities at the Center for Laboratory Animal Research of our institution for $1 \mathrm{wk}$ before any experiment to eliminate the stress effects of transportation and change in habitat. They were individually caged in a light-, temperature$\left(22 \pm 2^{\circ} \mathrm{C}\right)$, and humidity-controlled $(50 \pm 10 \%)$ environment with controlled feeding (normal pig chow; Ralston Purina Co., St. Louis, MO) and free access to water.

All procedures performed in this study were approved by the appropriate institutional guidelines and followed the American Heart Association guidelines for animal research.

After overnight fasting blood was withdrawn and platelets were labeled with ["1'Indium]tropolone $(300-400 \mu \mathrm{Ci})$ as we have previously described $(9,10)$. 18-24 h later the pigs were sedated with a mixture of ketamine and xylazine (Ketalar, Rompun) given intramuscularly, then intubated and ventilated with a Harvard respirator and 
anesthetized intravenously with sodium pentobarbital (Fort Dodge Lab., Fort Dodge, IA) with the minimal effective dose as previously described (4).

Through a left and right neck incision the carotid artery and jugular vein were isolated and catheterized. Blood was collected for baseline determination of hematocrit, platelet number, prothrombin time, and activated partial thromboplastin time (APTT). ${ }^{1}$ The animals were intravenously heparinized $(120 \mathrm{U} / \mathrm{kg}$ bolus plus continuous infusion of $100 \mathrm{U} / \mathrm{kg}$ per $\mathrm{h}$ for the duration of the study) and arterial blood was drawn into the original Plexiglas perfusion chamber by a peristaltic pump (model 7013; Master Flex, Cole-Palmer Instruments Co., Chicago, IL) at a controlled flow rate, and recirculated into the animal through the jugular vein. Blood samples were sequentially collected for APTT and heparin plasma level measurements (Coatest, heparin [S-2222]; KabiVitrum, Stockholm, Sweden). Baseline APTT levels were similar among the pigs and they were prolonged to over three times the control basal values after heparinization. The average prothrombin time ratio during the study was $1.49 \pm 0.016$. Average blood heparin levels during the perfusions were $1.37 \pm 0.036 \mathrm{U} / \mathrm{ml}$. Linear regression analysis of heparin levels during perfusion versus platelet deposition did not show any significant correlation: $y=57.4 x$ $+13.16(r=0.009 ; P=0.207 \mathrm{NS})$, where $y=$ platelet deposition $\times 10^{6} / \mathrm{cm}^{2}$ and $x=$ heparin levels (units/milliliter). Thus, the range of heparin levels reached with the heparin regime did not significantly affect platelet deposition results.

At the end of the study the animals were euthanized by an overdose of anesthetic.

Perfusion chamber. The tubular perfusion chamber (2) was designed to mimic the tubelike shape of the vasculature and has been fully characterized (3). In this chamber the vascular surface replaces a portion of the tubular wall that forms the blood channel and is directly exposed to the circulating blood.

A chamber with an internal diameter of $2.0 \mathrm{~mm}$ was selected and blood was perfused at a flow rate of $10 \mathrm{ml} / \mathrm{min}$. This flow condition in the laminar perfusion system gives a theoretically calculated local shear rate of $212 \mathrm{~s}^{-1}$. In some experiments blood was perfused at flow rates of 40 and $60 \mathrm{ml} / \mathrm{min}$ to mimic flow conditions in the coronary circulation. These flow conditions in the laminar perfusion system give local shear rates of 853 and $1,280 \mathrm{~s}^{-1}$, respectively. The local shear rates (in laminar flow conditions) of the study correspond to the physiological shear rates observed in patent large arteries and main branches found in different parts of the vasculature $(11,12)$. At these shear rates blood can be considered as a Newtonian fluid with constant viscosity $(13,14)$. The local shear rates were calculated from the expression for shear rate given for a Newtonian fluid in tube flow (15).

To quantitatively determine the influence of varying stenosis (nonparallel streamlines) on subsequent thrombosis, we modified the original chamber to simulate different degrees of stenosis, of the eccentric types as found in the coronary arteries of man $(16,17)$, and other arterial areas. The introduction of protruders of different sizes in the top part of the chamber creates a stenotic narrowing within the blood channel. Three stenoses, reducing the luminal flow channel by 35,55 , and $80 \%$ (in diameter) when the substrate was mounted, were studied in comparison with the $0 \%$ stenosis of the unobstructed laminar flow chamber (Fig. 1). In this study the length of the stenosis was constant at $4.5 \mathrm{~mm}$. This length has been reported as the average for single coronary stenosis in man (18). The extent and length of the induced stenosis were determined by measuring the remaining lumen of the channel once the substrate has been placed. The measurements were performed with a dissection microscope.

The changes in pressure across the chamber were measured (7754B system; Hewlett-Packard Co., Palo Alto, CA) both in parallel and nonparallel streamline flows ( $80 \%$ stenosis) at the flow rates used in the study (Table I).

1. Abbreviations used in this paper: ANOVA, analysis of variance; APTT, activated partial thromboplastin time.
Pig tunica media prepared from aortas harvested from normal untreated pigs was used as a model of severe arterial wall damage. The aortas were cleaned of the surrounding connective tissue, immediately deep-frozen in liquid $\mathrm{N}_{2}$, and stored at $-70^{\circ} \mathrm{C}$. Before the experiments the aortas were opened longitudinally and the intima with a thin portion of the subadjacent media was lifted up and peeled off starting from one corner (by retracting a raised corner). The major portion of the exposed media that remained overlying the adventitia was then divided into $30 \times 10-\mathrm{mm}$ segments to be placed in the chamber as previously described for deendothelialized vessel wall (1-3). Each aorta was randomly allocated to be exposed with a different stenosis.

Quantitation of platelet deposition. Autologous platelets were labeled with ["'In]tropolone according to our previously described technique (9). Platelets deposited on the perfused substrate $\left(\times 10^{6} / \mathrm{cm}^{2}\right)$ were determined by gamma well counting of the perfused matrix and normalized by blood activity (counts), platelet counts in blood, and surface area (1-4).

The axial dependence of platelet deposition with respect to the point of maximal stenosis was also analyzed. The fixed perfused specimens were oriented with respect to flow and divided into seven segments after perfusion. Segments 1 and 7, $1 \mathrm{~mm}$ in length (entrance and exit regions to the chamber), were discarded; the remaining five sections were $\sim 4.5 \mathrm{~mm}$ in length, with each corresponding to a different local flow profile. Segment 4 corresponded to the area of maximal stenosis (Fig. 1). These segments were individually counted in the gamma counter. Results in these experiments were expressed as deposited platelets $\left(\times 10^{5}\right)$ per unit (millimeters) length. Also, the axial percentage of distribution of deposited platelets was calculated.

Selected specimens were processed for optical morphometric evaluation and electron microscopy as previously described $(3,4)$.

Data analysis. Results were expressed as mean \pm 1 SEM unless otherwise stated. Statistical analysis was performed by $t$ test for paired or unpaired observations when groups had equal variances ( $F$ test) and by Mann-Whitney's $U$ test for groups with unequal variances ( $F$ test). Multiple group means were compared by one- or multiple-factor analysis of variance (ANOVA) with factorial or repeated measures analysis as pertinent.

\section{Results}

\section{Total platelet deposition on stenosed vessels}

\section{FLOW RATE $10 \mathrm{ML} / \mathrm{MIN}$}

Heparinized blood was perfused over stripped tunica media of normal pig aorta placed in the $2-\mathrm{mm}$ perfusion chamber for perfusion times of 1-30 min. Blood shear rate in 0\% stenosis (laminar blood flow) was $\sim 212 \mathrm{~s}^{-1}$ with a Reynold's number of 30 (4). Total platelet deposition on the perfused vascular wall (Fig. 2) (expressed as platelets $\times 10^{6} / \mathrm{cm}^{2}$ ) from $1-30 \mathrm{~min}$ of perfusion showed a statistically significant increase with perfusion time (ANOVA $P<0.003$ ). Hematocrit values $(26 \pm 0.3 \%)$ and whole blood platelet count $\left([398 \pm 10] \times 10^{3} / \mu \mathrm{l}\right)$ were similar in all perfusions $(n=92)$.

In perfusions lasting longer than $15 \mathrm{~min}$ a transitory drop in platelet deposition levels was detected, suggesting a partial dislodgment or lysis of the growing thrombus. However, the residual thrombus appeared to be extremely thrombogenic and able to grow rapidly to a significant thrombus (observe results at $30 \mathrm{~min}$ ).

The effects of three different degrees of stenosis on platelet-vessel wall interaction have been studied. The percent stenosis was calculated as $1-(A / B) \times 100$, where $A=$ luminal distance from the apex of stenosis to opposite wall, and $B$ $=$ luminal distance from wall to opposite wall proximal to the stenosis. This resulted in values of 35,55 , and $80 \%$ stenosis. The residual diameters at the apex of the stenosis were $1.3,0.9$, and $0.4 \mathrm{~mm}$, respectively. 


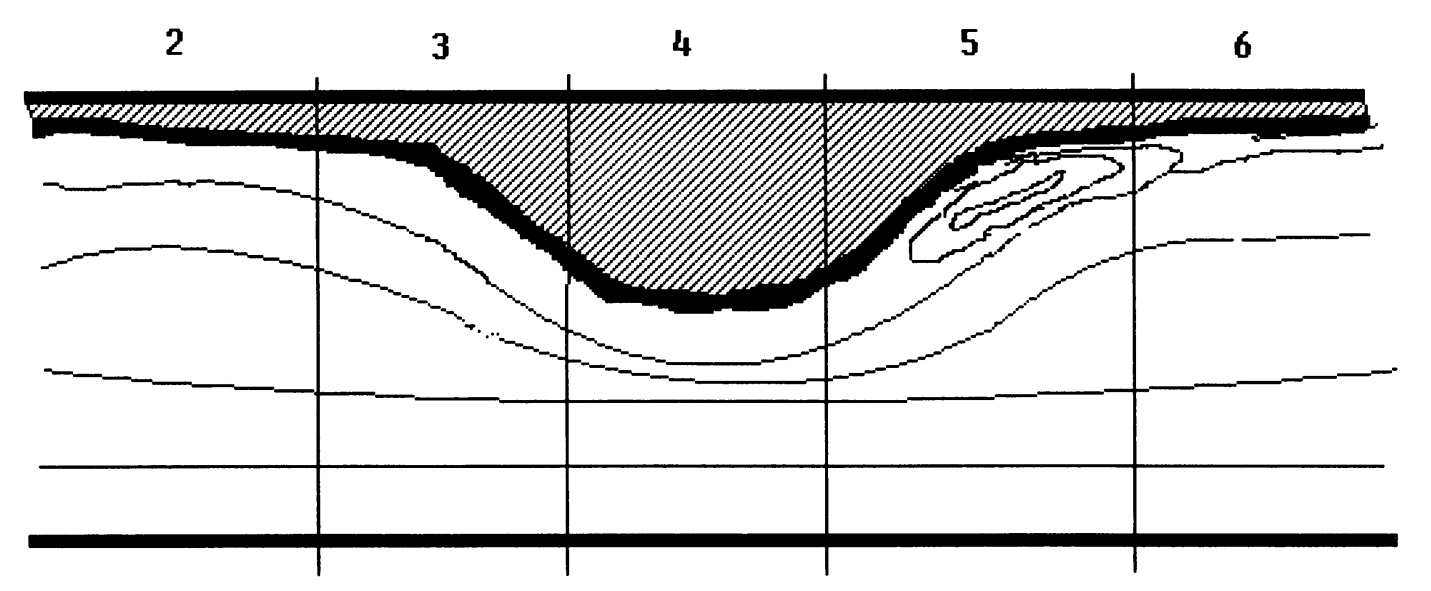

Figure 1. Diagram of a longitudinal section of the perfused tunica media mounted in the chamber with schematic flow streamlines. Segments 2-6 were divided and analyzed separately for axial dependence of platelet deposition. The blood flow was from left to right.

$35 \%$ stenosis. Blood was perfused over tunica media with a $35 \%$ stenosis located in segment 4 of the perfused vessel for perfusion times of 1-30 min. Total platelet deposition on the vessel $\left(\times 10^{6} / \mathrm{cm}^{2}\right)$ increased significantly with perfusion time (Fig. 2; $P=0.0001$ ). Perfusion times $>15$ min showed a transitory drop in platelet accumulation. Hematocrit $(26 \pm 0.7 \%, n=31)$ and platelet count $\left(392 \times 10^{3} \pm 13 \times 10^{3} / \mu \mathrm{l}\right.$, $n=31$ ) were similar in all perfusions. Total platelet deposition was significantly increased in the $35 \%$ stenotic vascular wall with respect to that in vascular wall with no stenosis (laminar flow conditions). Two-factor ANOVA ( $A=$ stenosis and $B$ $=$ perfusion time) showed significant effects for both factors individually and combined $(P<0.0001)$. There was no statistical difference between values of hematocrit (average $26 \pm 0.6 \%$ ) and whole blood platelet count (average [386 \pm 12$]$ $\left.\times 10^{3} / \mu \mathrm{l}\right)$ between both groups $(n=33)$.

$55 \%$ stenosis. Blood was perfused over tunica media with a localized $55 \%$ stenosis in segment 4 of the perfused vessel. Total platelet deposition on the perfused vessel (platelets $\left.\times 10^{6} / \mathrm{cm}^{2}\right)$ was significantly increased with perfusion time $(P$ $=0.001$; Fig. 2). Hematocrits (average $26 \pm 0.3 \%$ ) and whole blood platelet counts (average [409 \pm 14$] \times 10^{3} / \mu \mathrm{l}$ ) were similar in all perfusions $(n=39)$ at this flow condition.

$80 \%$ stenosis. Blood was perfused over stripped tunica media with a stenotic narrowing of $80 \%(n=53)$. Platelet deposition on the total exposed tissue (Fig. 2) increased significantly with perfusion time $(P=0.0019)$. Some reduction in platelet deposition could be measured from 20 min of perfusion (Fig. 2). Hematocrit $(26.4 \pm 0.3 \%)$ and whole blood platelet count $\left([378 \pm 19] \times 10^{3} / \mu \mathrm{l}\right)$ were similar in all perfusions.

HIGH FLOW RATES

Heparinized blood was perfused as before, but at higher flow rates to mimic flows typical of the coronary arteries.

Table I. Pressure Drop across the Perfusion Chamber ( $\mathrm{mmHg}$ )

\begin{tabular}{cccc}
\hline & \multicolumn{3}{c}{ Flow $(\mathrm{ml} / \mathrm{min})$} \\
\cline { 2 - 4 } Stenosis & 10 & 40 & 60 \\
\hline$\%$ & 2 & & \\
0 & 3 & 8 & 12 \\
80 & & 12 & 18 \\
\hline
\end{tabular}

Blood at a flow rate of $40 \mathrm{ml} / \mathrm{min}$ was perfused for 5,10 , and $30 \mathrm{~min}$. Blood shear rate in $0 \%$ stenosis (laminar parallel streamline flow) was $\sim 853 \mathrm{~s}^{-1}$ with a Reynold's number of 120. Total platelet deposition on the perfused vessel (Fig. 3) was significantly increased with respect to the results obtained at $10 \mathrm{ml} / \mathrm{min}(P=0.014$; Fig. 2$)$, but showed a similar profile increasing with perfusion time (ANOVA, $P<0.0006$ ). Hematocrit values $(25 \pm 0.3 \%)$ and whole blood platelet count $\left([355 \pm 13] \times 10^{3} / \mu \mathrm{l}\right)$ did not significantly change in the different perfusions of this group or with regard to the perfusions at a flow rate of $10 \mathrm{ml} / \mathrm{min}$. Heparinized blood was perfused over tunica media with 35 and $80 \%$ stenoses. Total platelet deposition on the vessel increased with perfusion time in both stenoses $(P=0.05$ and 0.002 , respectively; Fig. 3). Two-factor ANOVA of perfusions of flow rates of $40 \mathrm{ml} / \mathrm{min}$ showed significant effects on both perfusion time $(P=0.0002)$ and flow profile $(0,35$, and $85 \%$ stenosis; $P=0.01)$. Hematocrit and whole blood platelet count values for 35 and $80 \%$ stenosis were $25 \pm 0.5 \%$ and $324 \pm 10 \times 10^{3} / \mu \mathrm{l}$, and $25 \pm 0.4 \%$ and $359 \pm 16 \times 10^{3} / \mu \mathrm{l}$, respectively. There were no significant effects of hematocrit or platelet count on the platelet deposition values.

Blood at a flow rate of $60 \mathrm{ml} / \mathrm{min}$ was perfused for 5 and 10 min. Blood shear rate in $0 \%$ stenosis (laminar parallel-streamline flow) was $\sim 1,280 \mathrm{~s}^{-1}$ with a Reynold's number of 150 . Total platelet deposition was increased with respect to the platelet deposition levels obtained with lower flows, and there was again a significant dependence on perfusion time $(P$ $=0.006$; Fig. 3 ). When blood was perfused over tunica media with 35 and $80 \%$ stenoses, total platelet deposition also increased with perfusion time ( $P=0.003$ and 0.01 , respectively). Hematocrits and platelet counts were similar in all the perfusions of this group.

\section{Axial dependence of platelet deposition: distribution of accumulated platelets}

FLOW RATE $10 \mathrm{ML} / \mathrm{MIN}$

The axial dependence of platelet deposition was measured and compared by two-factor repeated measures ANOVA. There was a significant change in the percentage of distribution of the platelet deposition along the perfused surface, with deposition decreasing distally along the flow axis of the blood $(P<0.001$, factor B), but there was no significant change in the percent distribution with respect to the perfusion time (factor $A$ ). Therefore, the representative profile of the axial distribution of 

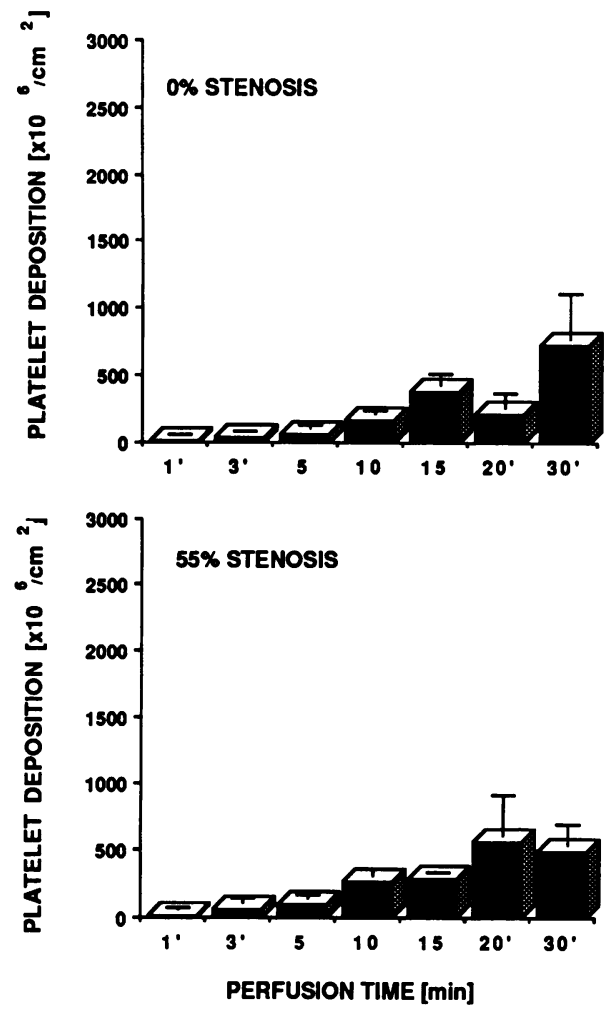
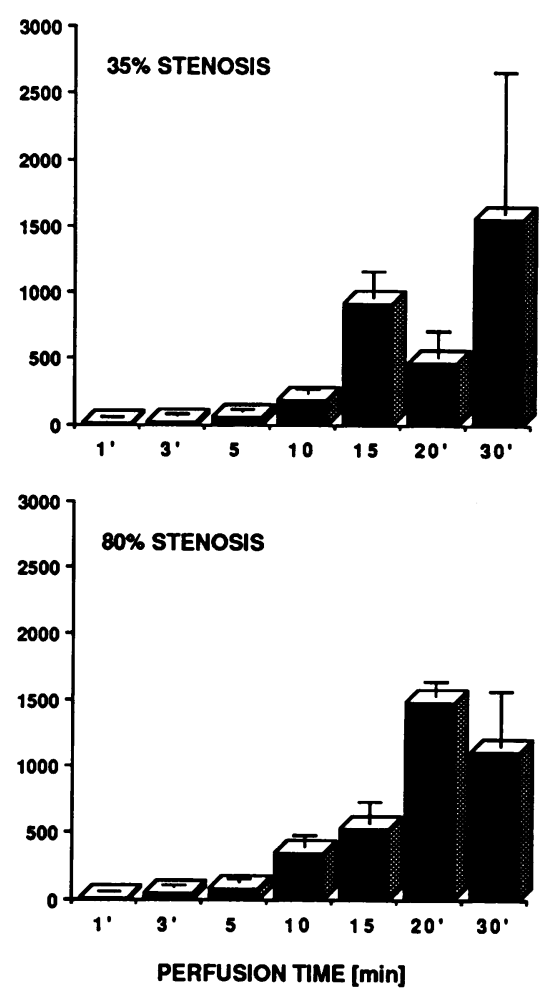

Figure 2. Mean values of total platelet deposition $\left(\times 10^{6} / \mathrm{cm}^{2} \pm 1\right.$ SEM $)$ are plotted versus perfusion time at various blood flow conditions (laminar, 35, 55, and $80 \%$ stenosis). These results are obtained when blood derived from heparinized pigs was perfused over tunica media at a flow rate of $10 \mathrm{ml} / \mathrm{min}$. platelets was reached as soon as 1 min of perfusion. Fig. 4 shows the average percent distribution of platelet deposition in laminar flow conditions ( $0 \%$ stenosis) from 1 to $30 \mathrm{~min}$ of perfusion.

The exact localization of the deposited platelets with respect to the apex of the $35 \%$ stenosis (segment 4 ) was analyzed (Fig. 4). The percent axial distribution of platelet deposition was significantly affected by the axial localization $(P$ $<0.0001)$. However, there was not a significant platelet accumulation on area 4 , peak of stenosis, over that on the other areas.

Comparing the percent axial distribution of platelets between 0 and $35 \%$ stenosis, we observed a significant increase in the number of platelets deposited on area 4 of the vessels with 35\% stenosis $(P<0.03)$.

When the axial effects of the $55 \%$ stenotic damaged vessel wall were analyzed, we observed that the distribution of platelets along the perfused surface was significantly $(P=0.001)$ modified with the axial position (one-factor ANOVA). Platelets significantly accumulated on the apex of the stenosis in each perfusion time (Scheffe F test, Fischer PLSD test). Twofactor repeated measures ANOVA showed significant effects for the axial position but not for perfusion time. Therefore, the average percent distribution of deposited platelets for all perfusion times is shown in Fig. 4. Comparison with the percent distribution of platelets in $0 \%$ stenosis showed that the distribution of platelets was significantly different in each segment $(P<0.001)$.

The axial distribution of platelet deposition (percentage) was found to change significantly with regard to the axial position at each perfusion time in the $80 \%$ stenosis studies. The apex of the stenosis (segment 4) showed the maximal accumulation of platelets (Fischer PLSD and Scheffe F test) in each perfusion time $(P<0.0001)$. When data were analyzed by two-factor ANOVA ( $A$, perfusion time; $B$, axial position) there was a significant change in the axial dependence (factor $\mathrm{A}, P$ $=0.0001$ ), but there was no significant effect on the perfusion time (factor A). Therefore, the results in percentages from 1 to 30 min have been pooled and presented in Fig. $4(n=35)$. The profile of platelet accumulation was reached in perfusion times as short as $1 \mathrm{~min}$. When compared with the percent distribution in laminar flow conditions, the differences in axial dependence were highly significant $(P<0.0001)$.

\section{HIGH FLOW RATES}

The axial dependence of platelet deposition was analyzed as before. Fig. 5 shows the average percent distribution of platelet deposition obtained at flow rates of 40 and $60 \mathrm{ml} / \mathrm{min}$. In laminar flow ( $0 \%$ stenosis) there was a slight but significant decrease in the percentage of platelet deposition along the flow axis of the perfused surface at $40 \mathrm{ml} / \mathrm{min}(P=0.003)$. However, in laminar flow ( $0 \%$ stenosis) at $60 \mathrm{ml} / \mathrm{min}$ there was no difference in the percentage of platelet deposition in the perfused segments (Fig. 5).

The exact localization of the deposited platelets with respect to the apex of the 35 and $80 \%$ stenoses (segment 4) was analyzed. The percentage axial distribution at a flow rate of $\mathbf{4 0}$ $\mathrm{ml} / \mathrm{min}$ was significantly affected by the axial localization at $35(P=0.009)$ and $80 \%(P=0.0001)$ stenoses. There was a significant accumulation on area 4 over that on the other areas at 35 and $80 \%$ stenoses (Fischer PLSD test; Fig. 5). At flow rate $60 \mathrm{ml} / \mathrm{min}$ the percent axial distribution was also significantly affected by axial localization at $35(P=0.004)$ and $80 \%(P$ $=0.0001$ ) stenoses. In both stenoses there was a highly significant accumulation of platelets in area 4, peak of the stenosis (Fischer PLSD test and Scheffe F test; Fig. 5). 
FLOW RATE 40 ML/MIN
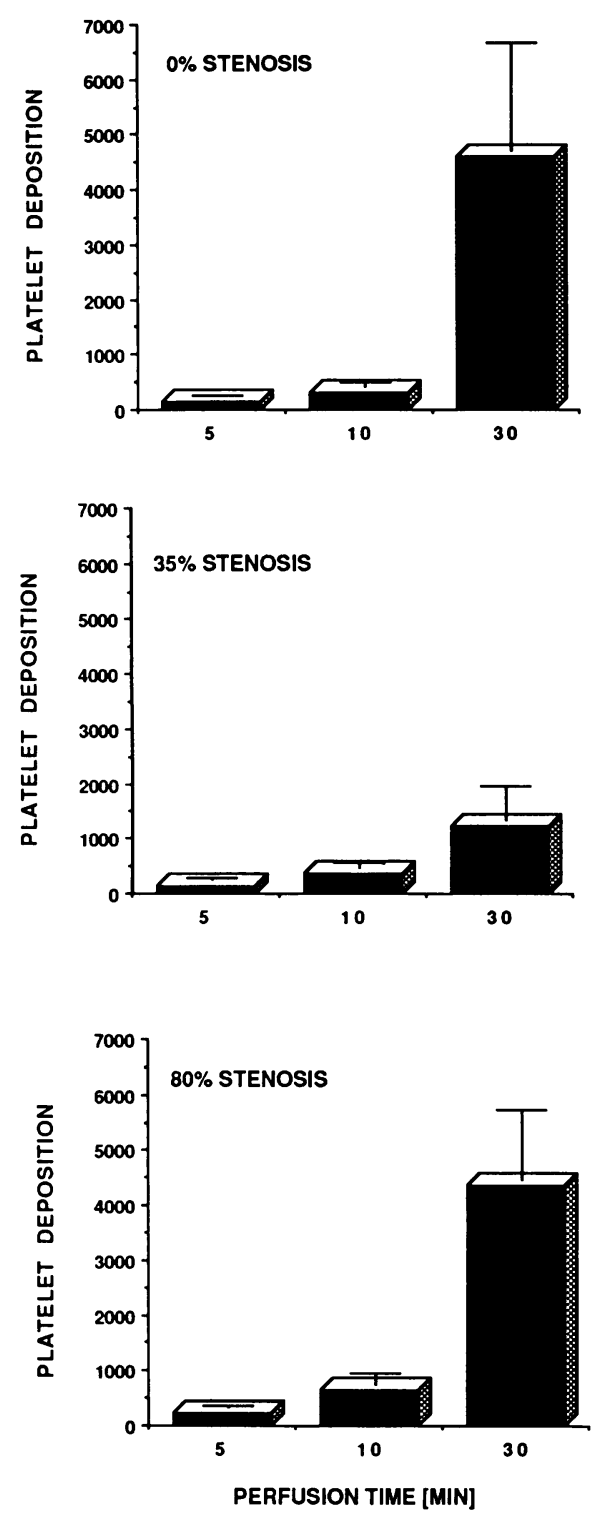

FLOW RATE 6OML/MIN
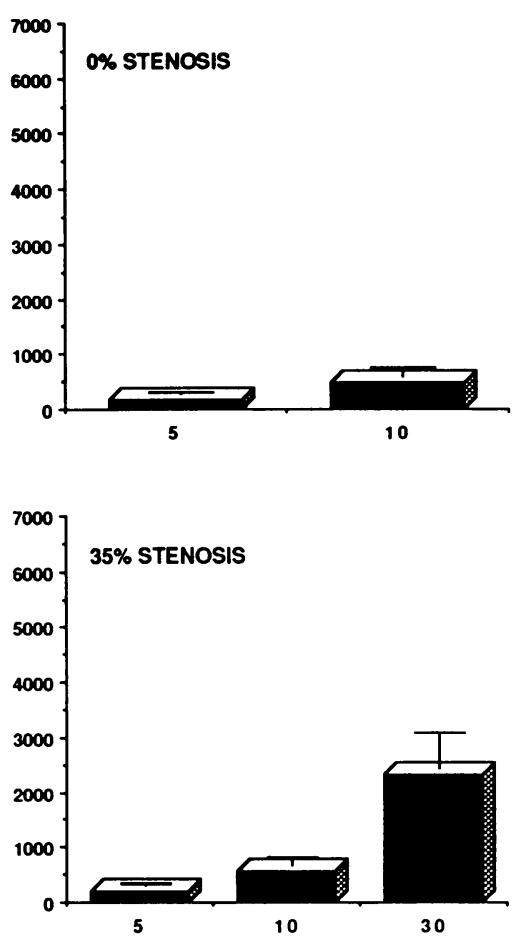

Figure 3. Mean values of platelet deposition $\left(\times 10^{6} / \mathrm{cm}^{2} \pm\right.$ SEM $)$ are plotted versus perfusion time at different blood flow conditions (laminar, 35 , and $80 \%$ stenosis). These results are obtained when blood derived from heparinized pigs was perfused over tunica media at flow rates of 40 and $60 \mathrm{ml} / \mathrm{min}$. Note the scalar change in the vertical axis to accommodate the increased platelet deposition compared with the lower flow rate in Fig. 2.
Axial dependence of platelet deposition: actual values of platelet deposition

FLOW RATE $10 \mathrm{ML} / \mathrm{MIN}$

When the axial distribution of platelet deposition was calculated as the number of platelets accumulated in each one of the five axially oriented sections (platelets $\times 10^{5} / \mathrm{mm}$ ), statistical analysis by two-factor repeated measure ANOVA showed that the local accruement of platelets increases significantly with the perfusion time $(P<0.0001)$. Fig. 6 shows the results of platelet deposition obtained at each perfusion time.

The analysis of the platelet deposition (platelets $\times 10^{5} /$ $\mathrm{mm}$ ) in each one of the axial segments in the perfusions with $35 \%$ stenosis showed a significant change in platelet accumulation with axial position $(P<0.0001)$, with perfusion time $(P$ $<0.0001)$, and with both factors combined $(P<0.0001)$. Fig. 6 shows the results obtained in each perfusion time. There was no significant platelet accumulation on the apex of the stenosis with regard to the other segments. When comparing the actual values of platelet deposition in the different axially oriented segments with those obtained in $0 \%$ stenosis, results were significantly higher in segments 2,3 , and 4 (peak; $P<0.0001$ ). Perfusion time also significantly influenced platelet deposition in each area $(P<0.0001)$.

Analysis of the actual values of platelet deposition in the axial segments of the vessels with 55\% stenosis showed that platelet numbers were significantly higher on segment 4 (Fischer PLSD, Scheffe F test) with respect to deposits in the other segments of the tissue $(2,3,5$, and 6$)$ at each perfusion time. Two-factor ANOVA ( $A$, perfusion time; $B$, axial position) showed significant effects of both factors on platelet deposition values (Fig. 6). When compared with the values of platelet deposition in the same segment in $0 \%$ stenosis (laminar flow conditions), segment 4 (peak of stenosis) showed significantly higher values of deposited platelets.

The number of platelets deposited in each axial segment varied with perfusion time and axial position $(P=0.0001)$, as well as for the combination of both factors $(P=0.0001)$ in $80 \%$ stenosis (Fig. 6). Segment 4 accrued a significantly higher 

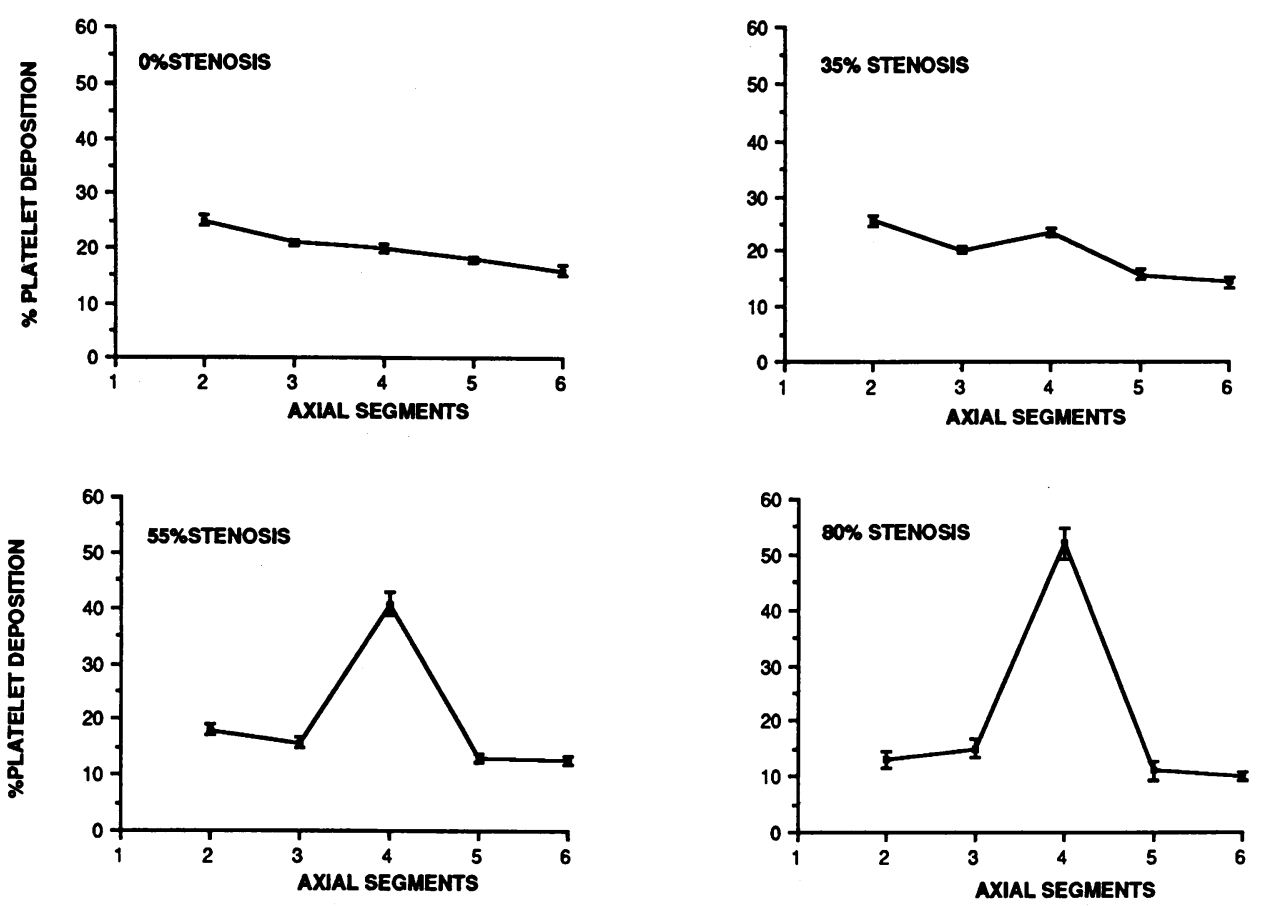

Figure 4. Axial distribution (in percentages) of platelet deposition along the axis of the blood flow. Segments 2-6 were separated according to the different local flow profiles. These results at laminar flow conditions ( $0 \%$ stenosis) and three different stenoses $(35,55$, and $80 \%$ ) were obtained when blood derived from heparinized pigs was perfused over tunica media at a flow rate of $10 \mathrm{ml} / \mathrm{min}$.

number of platelets $(P=0.001)$ than other segments of the tissue. When compared with the values of platelet deposition in $0 \%$ stenosis there was a significant increase due to the $80 \%$ stenosis $(P<0.0001)$.

\section{HIGHER FLOW RATES}

Actual numbers of platelets deposited in each one of the five axially oriented segments were calculated as before (Fig. 7). Platelet deposition increased with perfusion time for both flow rates, 40 and $60 \mathrm{ml} / \mathrm{min}$, as it did with the lower flow rate. At $40 \mathrm{ml} / \mathrm{min} 0$ and $35 \%$ stenoses did not show any significant platelet accumulation on the apex; however, $80 \%$ showed a significant accumulation of platelets of the apex of stenosis $P=0.0001$ ).

At a flow rate of $60 \mathrm{ml} / \mathrm{min} 0 \%$ stenosis did not show any difference in platelet deposition along the flow axis. However, 35 and $80 \%$ stenosis showed a highly significant accumulation of platelets in area $4(P=0.008$ and 0.0001 , respectively).

\section{Effect of the different stenotic narrowings on platelet deposition on the apex of the stenosis}

Analysis of the platelet deposition levels (platelets $\times 10^{5} / \mathrm{mm}$ ) by two-factor ANOVA on the apex (segment 4) of the stenosis ( $A$, stenosis; $B$, perfusion time) showed a very significant effect for both factors and their combination $(A, B$, and $A \times B, P$ $=0.0001$; Fig. 8). Also, the processes of thrombus dislodgment and recruitment occurred at the apex of the stenosis. When the same analysis was performed for the percentage of distribution data, results were statistically significant for stenosis $(P$ $=0.0001$ ) but not for perfusion time. Therefore, the representative average profile of percent distribution of platelet deposition in the apex of the stenosis was reached as soon as $1 \mathrm{~min}$ of perfusion.

The same analysis was applied to flow rates of 40 and 60 $\mathrm{ml} / \mathrm{min}$. At both flow rates we observed significant effects on platelet deposition on the apex of both stenosis $(A, 0.003$ and
0.03 , respectively) and perfusion time $(B, 0.003$ and 0.0007 , respectively).

\section{Discussion}

In medium to large arteries there is a clear association of thrombosis with atherosclerosis. Extensive, clinically relevant thrombus formation occurs mainly in regions where there is stenotic narrowing of the lumen (19). However, the role of blood flow and specifically the blood shear rate and shear stress in determining thrombosis on surfaces has been investigated primarily in laminar flow conditions in which flow streamlines are parallel. Whereas flow in normal, nondiseased vessels may be approximated by such perfusion systems, the flow patterns in stenotic-diseased vessels are complex and not characterized by parallel streamlines. A number of modeling studies (engineering) have been directed at quantitatively describing the patterns characteristic of flow in stenotic vessels (20). There is a convective transport to the wall by which the corpuscles in the flow stream (cells) are carried toward the wall along curved streamlines having pronounced radial velocity components. Such flow patterns found in regions of disturbed flow as those encountered at stenoses are neither laminar nor turbulent. In these areas secondary flow motions develop in directions away from the primary flow, and often there is separation of the streamlines from the vessel wall, with the formation of a vortex or a recirculation zone between the forward flowing mainstream and the walls.

In the present study we analyzed the effects of various degrees of eccentric stenoses on platelet interaction with severely damaged vessel wall. This is the first report of a controlled study of thrombus formation in stenotic flow conditions analyzing the local distribution of platelet deposition (axial distribution). This axial dependence analysis of platelet thrombus formation was performed with regard to the apex of 
FLOW RATE 4OMLMIN
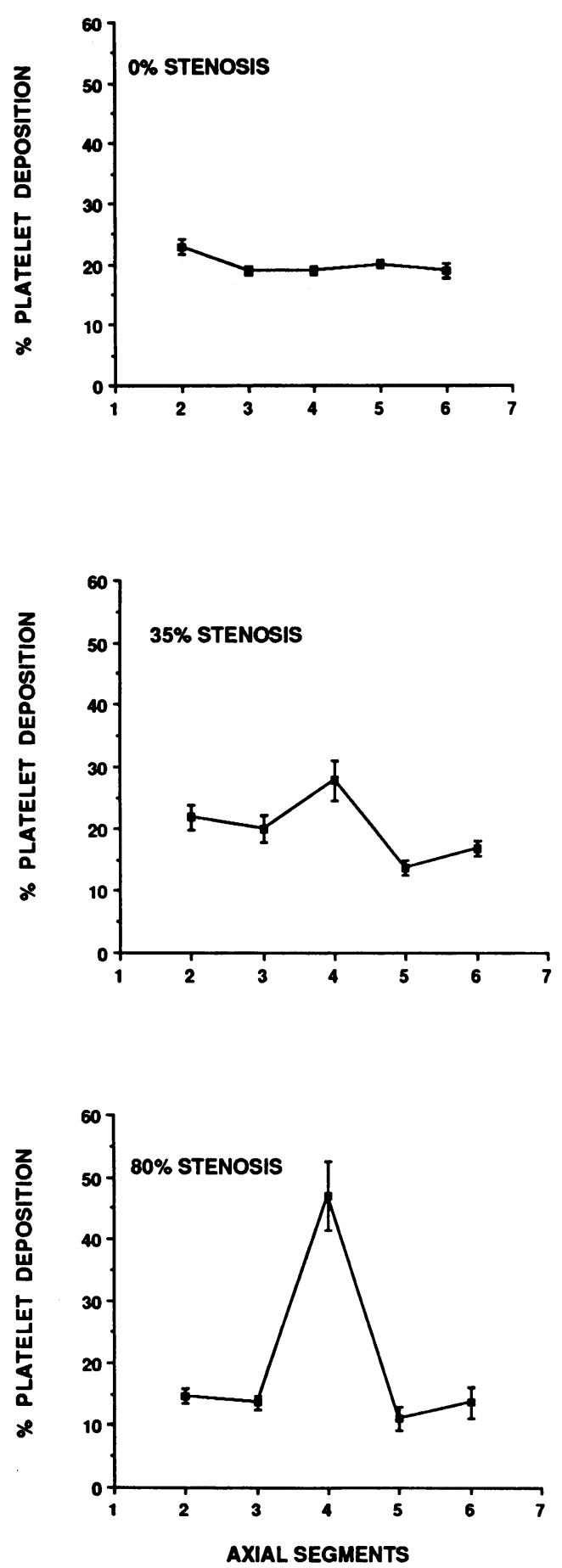

FLOW RATE 6OMLMIN
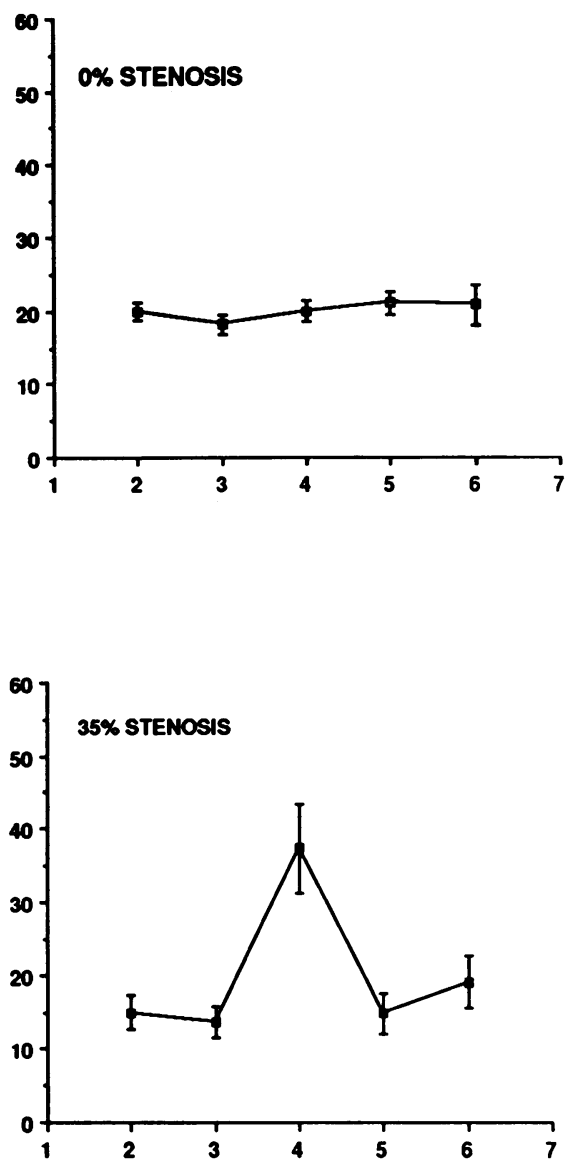

Figure 5. Axial distribution (in percentages) of platelet deposition along the axis of the blood flow. Segments 2-6 were separated according to the different local flow profiles. These results at laminar flow conditions ( $0 \%$ stenosis) and two different stenoses ( 35 and $80 \%$ ) were obtained when blood derived from heparinized pigs was perfused over tunica media at flow rates of 40 and $60 \mathrm{ml} / \mathrm{min}$. the stenosis and according to the changes in local flow conditions.

We used stripped tunica media as a model of deep vessel injury. This is a highly thrombogenic material that induces thrombus formation also in laminar flow conditions at any shear rate and perfusion time. This surface, consisting of fibrillar native collagens (types III and I), proteoglycans, glycosaminoglycans, elastin, and smooth muscle cells (with surfaceexposed tissue factor), is exposed in the ruptured atheroscle- rotic wall, although probably in different relative proportions with respect to the normal vessel wall (21).

Even in the absence of stenosis (laminar flow), platelet deposition on severely damaged vessel wall (tunica media) increased significantly with perfusion time. The distribution of platelets on the surface in laminar flow at shear rates of $212 \mathrm{~s}^{-1}$ followed the mass transfer theory and was distally decreased along the flow axis of the blood (Fig. 4), supporting the theory of platelet depletion from the boundary layer of the blood flow 

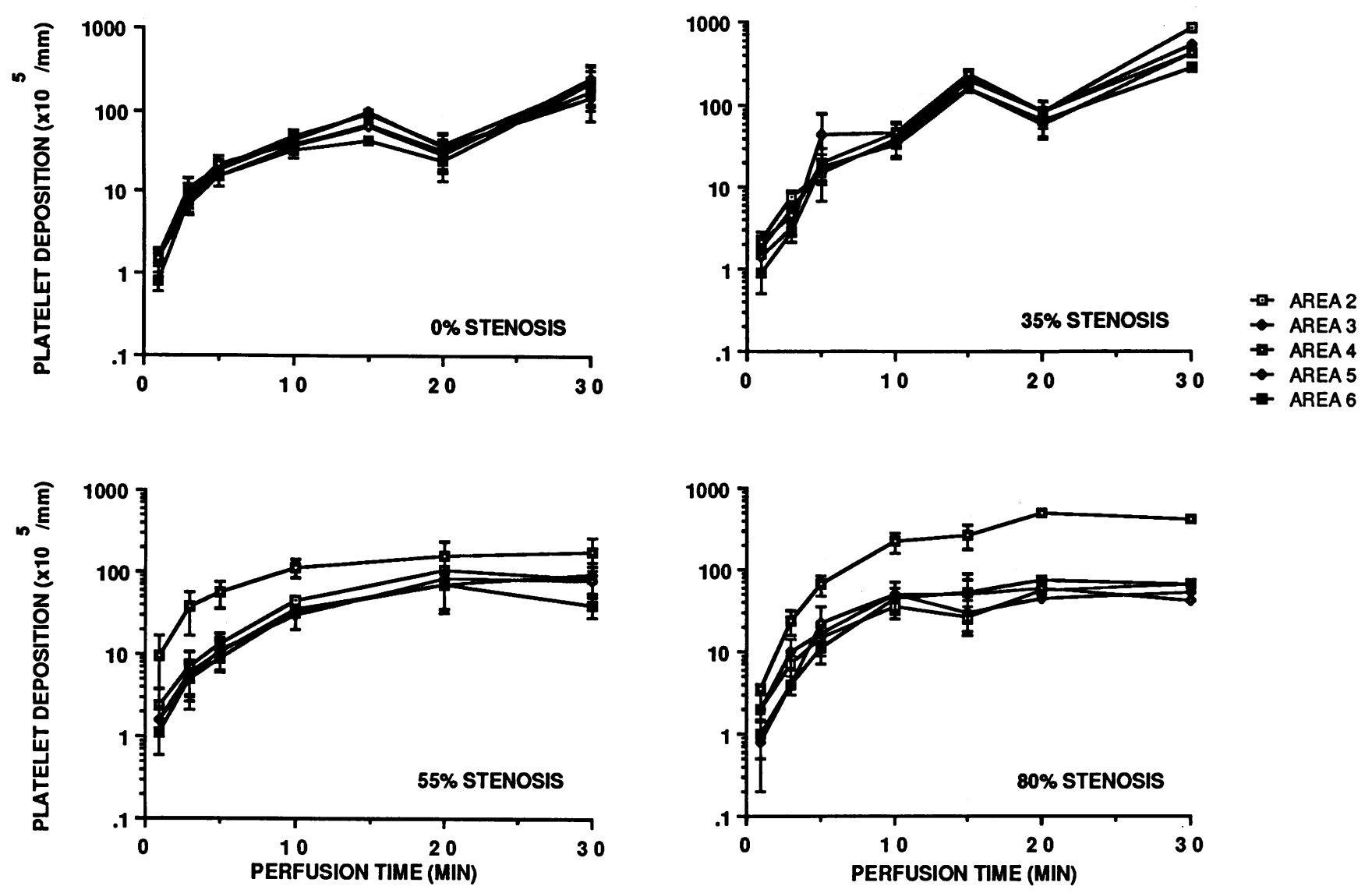

Figure 6. Values of platelet deposition $\left(\times 10^{5} / \mathrm{mm} \pm 1 \mathrm{SEM}\right)$ on the individual axial segments along the perfusion time. Blood was perfused at a flow rate of $10 \mathrm{ml} / \mathrm{min}$.

$(22,23)$. A similar pattern of distribution was reported in platelet deposition on collagen-coated surfaces (24). At a shear rate of $850 \mathrm{~s}^{-1}$ the distribution of platelets decreasing distally along the flow axis was less marked but still significant. However, at a shear rate of $1,250 \mathrm{~s}^{-1}$ platelet distribution was uniform along the flow axis (Fig. 5). Blood flow rates of 40 and 60 $\mathrm{ml} / \mathrm{min}$ induced high shear rate conditions in which very significant thrombus growth rates occurred even in laminar flow $\left(26 \times 10^{6}\right.$ and $33 \times 10^{6}$ platelets $/ \mathrm{cm}^{2} \cdot \mathrm{min}^{-1}$, respectively, at 5 min of perfusion). A blood flow rate of $10 \mathrm{ml} / \mathrm{min}$ induced a thrombus growth rate of $13 \times 10^{6}$ platelets $/ \mathrm{cm}^{2} \cdot \mathrm{min}^{-1}$ at 5 min of perfusion.

At any flow rate total platelet deposition increased with the increasing degrees of stenosis from 0 to $80 \%$ of occlusion. However, platelet accumulation on the apex of the stenosis (segment 4 ) with regard to the areas in its vicinity (segments 2 , 3,5 , and 6) was significantly increased when the stenosis was $>55 \%$ at a flow rate of $10 \mathrm{ml} / \mathrm{min}$. At flow rates of 40 and 60 $\mathrm{ml} / \mathrm{min}$ significant increases in platelet deposition on the apex were already observed with a $35 \%$ stenosis. An increase in flow rate without modification of geometry produces an increase in shear rate and we have previously shown that platelet deposition increases with shear rate on thrombogenic surfaces $(2,3$, $5)$. A small narrowing ( $35 \%$ ) on a geometry with a shear rate of 850 or $1,250 \mathrm{~s}^{-1}$ induces a more potent stimulus for platelet activation and deposition than the same narrowing at a local shear rate of $212 \mathrm{~s}^{-1}$.
At a flow rate of $10 \mathrm{ml} / \mathrm{min}$ when the stenosis was $35 \%$ there was a slightly higher accumulation of platelets on the apex of the stenosis (segment 4); however, in 55 and $80 \%$ the percentage of platelets accumulated on the apex was significantly higher than in any other area of the perfused substrate. At flow rates of 40 and $60 \mathrm{ml} / \mathrm{min}$ the accumulation in segment 4 was significantly greater than in the other segments already at $35 \%$ stenosis. Hence, platelets accumulate on top of the stenosis, not in the area of impact (proximal to stenosis, segment 3) or vortex (segment 5). It is interesting that this distribution was already attained at $1 \mathrm{~min}$ of perfusion. When the deposition on segment 4 was analyzed at different perfusion times and percentages of stenosis, there was a significant effect of time, stenosis, and the combination of both factors in thrombus growth.

Upon plaque rupture and exposure of intraplaque thrombogenic material to blood flow the residual remodeled plaque will influence the luminal area; hence, the sudden change in local flow factors and the substrate reactivity will increase the likelihood of an acute thrombotic occlusion.

Cell wall encounters arise from the continual shear-induced collisions between red cells that lead to lateral displacements of their paths as well as those of white cells and platelets. Thus, cells flowing in the periphery of vessels are frequently displaced to the wall (20). The hematocrit has, therefore, a very important role in cell-wall interaction. In our studies the hematocrit was similar in all the experiments and cannot be 
FLOW RATE 4OMLMIN
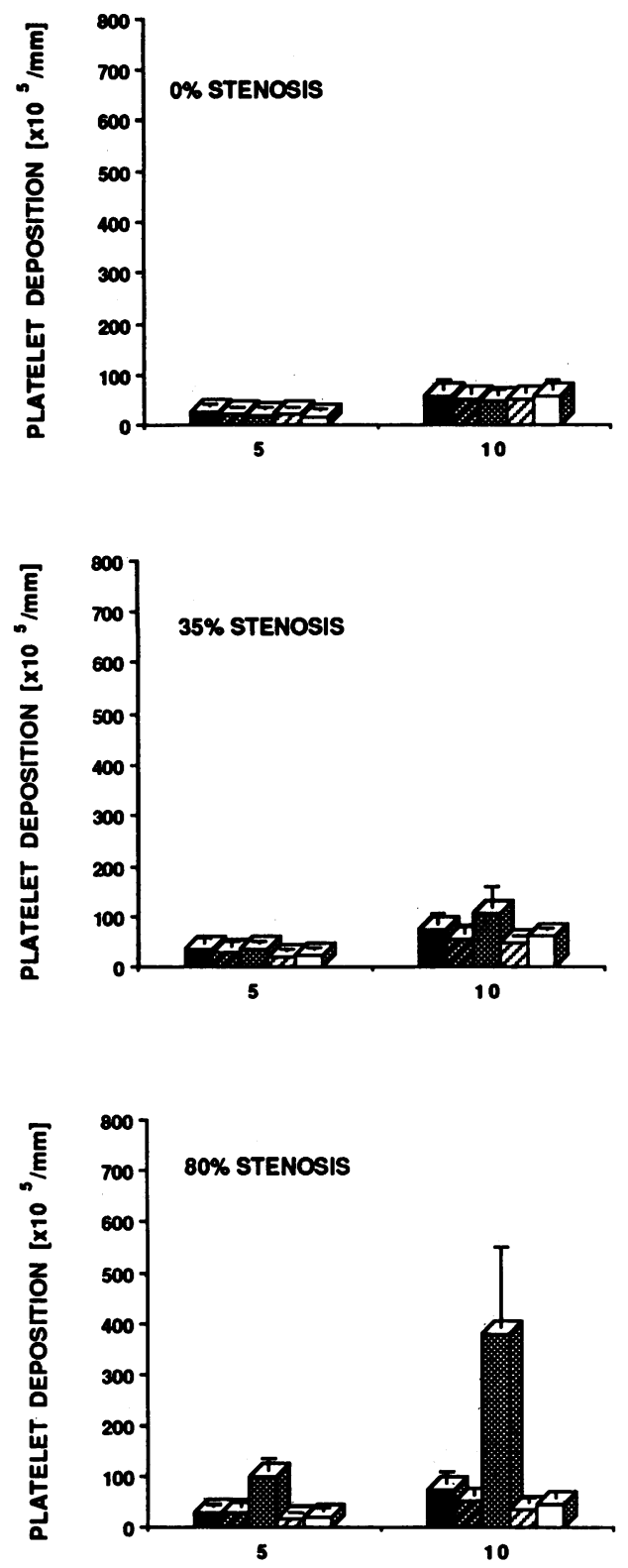

PERFUSION TIME [MIN]
FLOW RATE 60MLINN
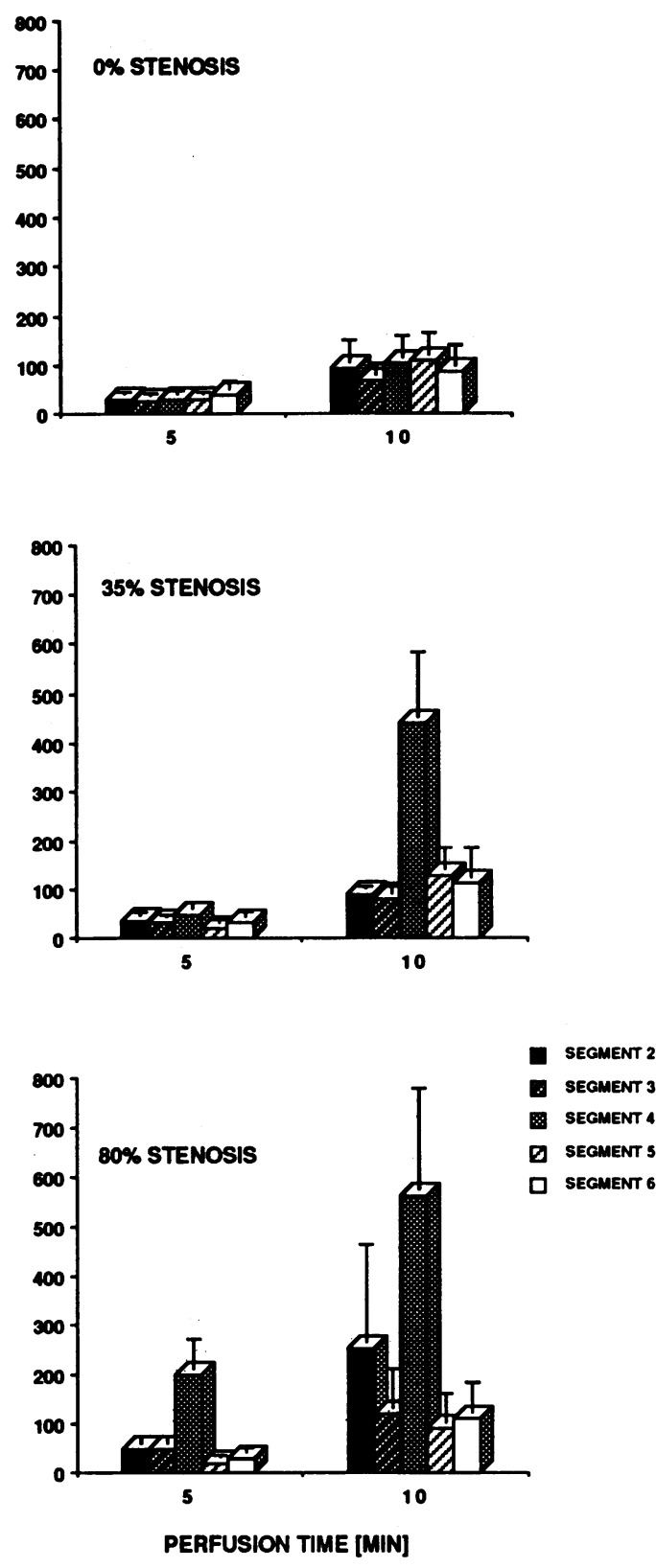

Figure 7. Values of platelet deposition $\left(\times 10^{5}\right.$ / $\mathrm{mm} \pm 1 \mathrm{SEM}$ ) on the individual axial segments at different perfusion times. Blood was perfused at flow rates of 40 and 60 $\mathrm{ml} / \mathrm{min}$. considered an interassay variable that may have influenced our platelet deposition results at the different stenotic conditions.

Finally, as shown in Figs. 2 and 8, when perfusions lasted longer than $15 \mathrm{~min}$ we observed a transient decrease in platelet deposition, suggesting that removal predominated over deposition. Thus, masses of platelets must have been dislodged from the substrate. This partial embolization left behind an extremely reactive surface able to recover a highly significant number of platelets quite rapidly. This embolization and regrowth phenomena are currently being studied in our laboratory by continuous imaging. This regrowth is a finding that may explain the increased risk of reocclusion after thrombolysis in cases with angiographically demonstrable residual coro- nary thrombus on stenoses $(25,26)$. Our experimental observations suggest that in addition to the local flow characteristic in the reperfused area, a surface consisting of a partially disrupted thrombus can have thrombogenic properties of its own.

At long perfusion times and flow rates of 10 and $40 \mathrm{ml} / \mathrm{min}$ we have measured less accumulation of platelets in 35 or $55 \%$ stenosis than in $0 \%$ (Figs. 2 and 3). Those results could be a consequence of the above-mentioned phenomenon, the tendency of the thrombus to transiently embolize and regrow. Since the methodology applied in the study requires a stoppage of the blood perfusion to measure platelet deposition (end point analysis), we stop the process without the knowledge of the dynamic status of the thrombus. If embolization or re- 

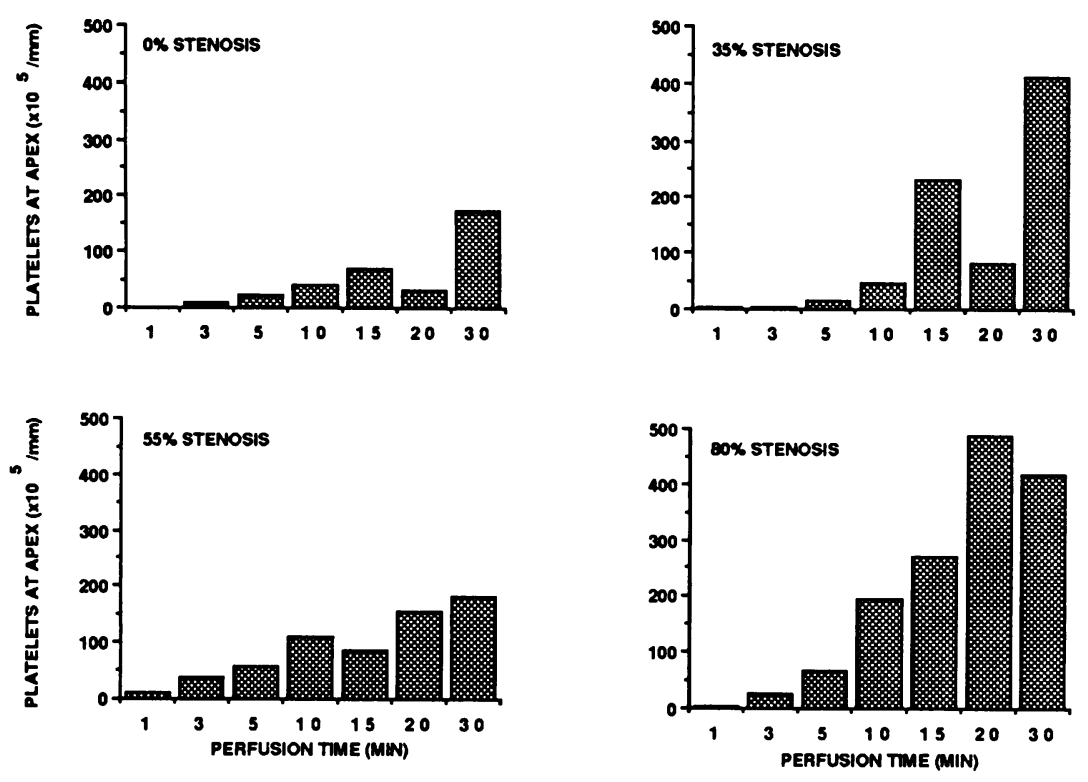

Figure 8. Average value of platelet deposition $\left(\times 10^{5} / \mathrm{mm}\right)$ on the apex of the stenosis along the perfusion time (ANOVA; $A$, stenosis; $P=0.0001$; $B$, perfusion time; $P=0.0001$ ) at a flow rate of 10 $\mathrm{ml} / \mathrm{min}$.

growth is occurring the results will be substantially affected. In fact, in perfusions $>15 \mathrm{~min}$ there is an increase in variability about the mean (Figs. 2 and 3).

The formation of a thrombus is a complex process regulated by flow that involves the formed elements of blood and also the different components of the injured vessel wall as well as the plasma coagulants. The accurate understanding of the underlying phenomena in cell-vessel wall and cell-cell interaction in flowing blood conditions, instead of in static assays, should be the basis for future investigation regarding prevention and management of thrombotic events.

\section{Acknowledgments}

The authors thank Dr. V. Fuster for his expert comments during the revision of the manuscript and for his continuous support and collaboration. The authors are also grateful to Dr. V. Turitto and Dr. M. Cohen for their advice.

This study was supported by U. S. Public Health Service grant HL-39840.

\section{References}

1. Goldsmith, H. L., and T. Karino. 1987. Interactions of human blood cells with the vascular endothelium. Ann. NY Acad. Sci. 516:468-483.

2. Badimon, L., J. J. Badimon, A. Galvez, J. H. Chesebro, and V. Fuster. 1986. Influence of arterial damaged and wall shear rate on platelet deposition: ex vivo study in a swine model. Arteriosclerosis. 6:312-320

3. Badimon, L., V. T. Turitto, J. A. Rosemark, J. J. Badimon, and V. Fuster. 1987. Characterization of tubular flow chamber for studying platelet interaction with biological and prosthetic materials. J. Lab. Clin. Med. 6:706-718.

4. Badimon, L., J. J. Badimon, V. T. Turitto, J. Rand, and V. Fuster. 1987. Platelet deposition on von Willebrand factor deficient vessels. J. Lab. Clin. Med. 5:634-647.

5. Badimon, L., J. J. Badimon, V. T. Turitto, S. Vallabhajosula, and V. Fuster. 1988. Platelet thrombus formation on collagen type I: a model of deep vessel injury. Influence of blood rheology, von Willebrand factor and blood coagulation. Circulation. 78:1431-1442.
6. Steele, P. M., J. H. Chesebro, A. W. Stanson, D. R. Holmes, Jr., M. K. Dewanjee, L. Badimon, and V. Fuster. 1985. Balloon angioplasty: natural history of the pathophysiologic response to injury in pig model. Circ. Res. 57:105-112.

7. Lam, J. Y. T., J. H. Chesebro, P. M. Steele, M. K. Dewanjee, L. Badimon, and V. Fuster. 1986. Deep arterial injury during experimental angioplasty: relationship to a positive ${ }^{111}$ Indium-labelled platelet scintigram, quantitative platelet deposition, and mural thrombosis. $J$. Am. Coll. Cardiol. 8:1380-1386.

8. Lam, J. Y. T., J. H. Chesebro, P. M. Steele, L. Badimon, and V. Fuster. 1987. Is vasospasm related to platelet deposition? Relationship in a porcine preparation of arterial injury in vivo. Circulation. 75:243-248.

9. Vallabhajosula, S., J. Machac, S. J. Goldsmith, H. Lipszyc, L. Badimon, J. Rand, and V. Fuster. 1986. Indium-111 platelet kinetics in normal human subjects. J. Nucl. Med. 27:1669-1674.

10. Badimon, L., V. Fuster, J. H. Chesebro, and M. K. Dewanjee. 1983. New "ex vivo" radioisotopic method of quantitation of platelet deposition: studies in four animal species. Thromb. Haemostasis. 50:639-644.

11. Goldsmith, H. L., and V. T. Turitto. 1986. Rheological aspect of thrombosis and hemostasis. Thromb. Haemostasis. 55:415-435.

12. Whitmore, R. L. 1968. Rheology of the Circulation. Pergamon Press, New York. 888.

13. Merrill, E. W. 1969. Rheology of blood. Physiol. Rev. 49:863-

14. Levich, V. G. 1962. Physicochemical Hydrodynamics. Prentice-Hall, Englewood Cliffs, NJ.

15. Bird, R. B., W. E. Steward, and E. N. Lightfoot. 1960. Transport Phenomena. John Wiley \& Sons, New York. 42-47.

16. Ambrose, J. A., S. L. Winters, R. R. Arora, J. I. Haft, J. Goldstein, K. P. Rentrop, R. Gorlin, and V. Fuster. 1985. Coronary angiographic morphology and myocardial infarction: a link between the pathogenesis of unstable angina and myocardial infarction. J. Am. Coll. Cardiol. 6:1233-1238.

17. Fuster, V., L. Badimon, M. Cohen, J. A. Ambrose, J. J. Badimon, and J. Chesebro. 1988. Insights into the pathogenesis of acute ischemic syndromes. Circulation. 1213-1220.

18. Meier, B., A. Gruentzig, J. Hollman, T. Ischinger, and J. M. Bradford. 1983. Does length or eccentricity of coronary stenoses influence the outcome of transluminal dilatation? Circulation. 3:497-499. 
19. Fuster, V., J. H. Chesebro, J. J. Badimon, and L. Badimon. 1987. Coronary artery disease, platelets and thrombosis. In Hemostasis and Thrombosis. R. W. Colman, J. Hirsh, V. J. Marder, and E. W. Salzman, editors. J. B. Lippincott Co., Philadelphia. 1290-1300.

20. Karino, T., H. L. Goldsmith, M. Motomiya, S. Mabuchi, and Y. Sohara. 1987. Flow patterns in vessels of simple and complex geometries. Ann. NY Acad. Sci. 516:422-441.

21. Barnes, M. J. 1985. Collagens in atherosclerosis. Collagen Relat. Res. 5:65-97.

22. Turitto, V. T., and H. R. Baumgartner. 1975. Platelet deposition on subendothelium exposed to flowing blood: mathematical analysis of physical parameters. Trans. Am. Soc. Artif. Intern. Organs. 21:593-600.

23. Turitto, V. T., H. J. Weiss, and H. R. Baumgartner. 1979.
Rheological factors influencing platelet interaction with vessel surfaces. J. Rheol. 23:735-749.

24. Sakariassen, K. S., and H. R. Baumgartner. 1989. Axial dependance of platelet collagen interactions in flowing blood: upstream thrombus growth impairs downstream platelet adhesion. Arteriosclerosis. 9:33-42.

25. Ellis, S. G., G. S. Roubin, S. B. King, J. S. Douglas, W. S. Weintraub, R. G. Thomas, and W. R. Cox. 1988. Angiographic and clinical predictors of acute closure after native vessel coronary angioplasty. Circulation. 77:372-379.

26. Brown, B. G., C. A. Gallery, R. S. Badger, J. W. Kennedy, M. D. Mathey, E. L. Bolson, and H. T. Dodge. 1986. Incomplete lysis of thrombus in the moderate underlying atherosclerotic lesion during intracoronary infusion of streptokinase for acute myocardial infarction. Circulation. 73:653-661. 